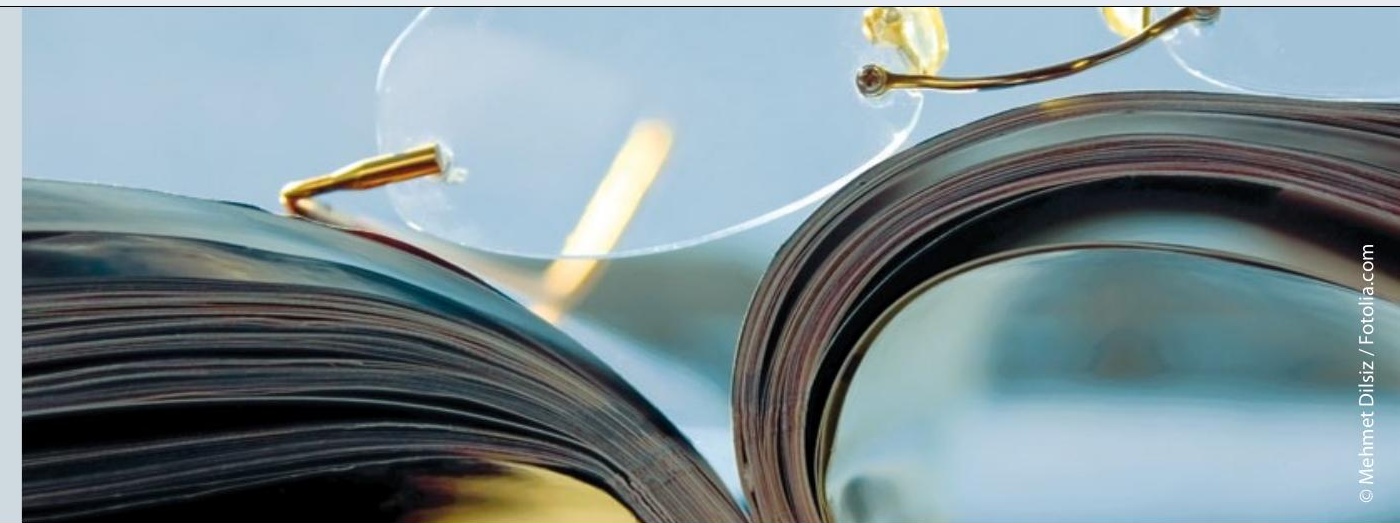

\section{KVT der Depression auch am Telefon wirksam!}

Gerade in ländlichen Gegenden kann der Weg zum Therapeuten das größte Hindernis bei der Aufnahme einer Psychotherapie sein. Ruf doch mal an dieses Motto könnte hier Abhilfe schaffen, denn die Therapie am Telefon steht der im Angesicht des Therapeuten hinsichtlich der Wirksamkeit in nichts nach.

E in Forscherteam aus Chicago untersuchte an über 300 Patienten mit Depression, ob es Unterschiede hinsichtlich der Therapieadhärenz und der Wirksamkeit einer kognitiven Verhaltenstherapie (KVT) am Telefon verglichen mit der Behandlung im direkten Kontakt gibt. Dafür erhielten alle Patienten 20 Therapiesitzungen, wobei per Zufall entschieden wurde, auf welchem Weg die Behandlung den Patienten erreichen sollte. Die Wirksamkeit der Behandlung wurde durch validierte Skalen (Hamilton Depressionskala und Patient Health Questionaire) überprüft, die von Ratern, die über den Weg der Behandlung nicht informiert waren, erhoben wurden. Bei den telefonisch behandelten Patienten zeigte sich eine geringere Abbrecherquote (circa $20 \%$ verglichen mit circa $30 \%$ in der "face-to-face" Gruppe), wobei dieser Unterschied in den ersten Sitzungen entstand. Die Wirksamkeit der Therapie zeigte sich in beiden Gruppen, ohne dass signifikante Unterschiede zwischen dem Weg der Behandlung festgestellt werden konnten. In einer Katamneseuntersuchung nach sechs Monaten zeigten sich jedoch die Patienten, die die klassische Therapie erhalten hatten, weniger depressiv als die Patienten aus der Telefongruppe.

Kommentar: Diese methodisch auf höchstem Niveau durchgeführte randomisiertkontrollierte Psychotherapiestudie zeigt, dass die Behandlung per Telefon gut funktioniert und damit dort, wo es Barrieren auf dem Weg zum Therapeuten gibt, dazu führen kann, dass Patienten die Therapie zu Ende führen. Der längerfristige Vorteil des klassischen Behandlungssettings lässt sich damit erklären, dass weniger motivierte Patienten in der Telefongruppe länger durchgehalten haben, danach aber rascher wieder einen Rückfall erlitten. Die Ergebnisse der Studie könnten eine große Bedeutung für die psychotherapeutische Versorgung depressiver Menschen in ländlichen
Gegenden gewinnen, allerdings müssten dafür die passenden Strukturen geschaffen werden. Interessant dürfte sein, welchen Einfluss weitere telemedizinische Möglichkeiten der Psychotherapie haben, zum Beispiel über das Internet.

Prof. Dr. Helge Frieling

Mohr DC et al. Effect of Telephone-Administered vs Face-to-face Cognitive Behavioral Therapy on Adherence to Therapy and Depression Outcomes Among Primary Care Patients - A Randomized Trial. JAMA 2012; 307: 2278-85

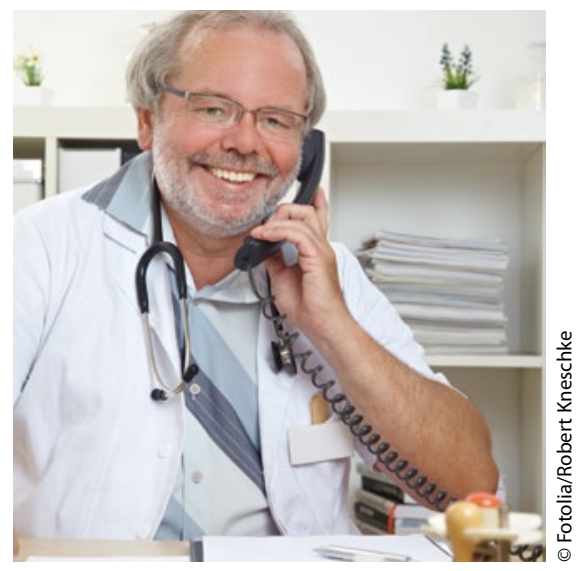

Psychotherapie am Telefon kann helfen

eine Therapie mit Escitalopram und $5 \mathrm{~g}$ Kreatin und 27 Frauen Escitalopram und Placebo. Hauptzielkriterium war das Ansprechen auf die Therapie, welches als ein Rückgang des Punktewertes auf der Hamilton-Skala (HAMD) um $50 \%$ definiert wurde. Weitere Kriterien waren die Zeit bis zum Ansprechen auf die Therapie, eine Remission (HAMD $<7$ Punkte) und die Häufigkeit von Nebenwirkungen. Patienten mit Kreatinaugmentation sprachen in der Studie früher und stärker auf die 